\title{
The cryptic ushA gene (ushAc) in natural isolates of Salmonella enterica (serotype Typhimurium) has been inactivated by a single missense mutation
}

\author{
David Innes, ${ }^{1}$ Ifor R. Beacham, ${ }^{2}$ Carie-Anne Beven, ${ }^{2}$ Meaghan Douglas, ${ }^{1}$ \\ Michael W. Laird, John C. Joly ${ }^{3}$ and Dennis M. Burns ${ }^{1}$
}

Author for correspondence: Dennis M. Burns. Tel: +6173875 5069. Fax: +617 38757773. e-mail: D.Burns@sct.gu.edu.au

1 School of Biomolecular and Biomedical Science, Griffith University, Brisbane, Qld 4111, Australia

2 School of Health Science, Griffith University, PMB 50 Gold Coast Mail Centre, Gold Coast, Qld 4217, Australia

3 Genentech Inc., 1 DNA Way, South San Francisco, CA 94080 , USA

\begin{abstract}
Two mutational mechanisms, both supported by experimental studies, have been proposed for the evolution of new or improved enzyme specificities in bacteria. One mechanism involves point mutation(s) in a gene conferring novel substrate specificity with partial or complete loss of the original (wild-type) activity of the encoded product. The second mechanism involves gene duplication followed by silencing (inactivation) of one of these duplicates. Some of these 'silent genes' may still be transcribed and translated but produce greatly reduced levels of functional protein; gene silencing, in this context, is distinct from the more common associations with bacterial partitioning sequences, and with genes which are no longer transcribed or translated. Whereas most Salmonella enterica strains are ushA ${ }^{+}$, encoding an active $5^{\prime}$-nucleotidase (UDP-sugar hydrolase), some natural isolates, including most genetically related strains of serotype Typhimurium, have an ushA allele (designated ush $A^{c}$ ) which produces a protein with, comparatively, very low 5'nucleotidase activity. Previous sequence analysis of cloned ushAc and ushA+ genes from serotype Typhimurium strain LT2 and Escherichia coli, respectively, did not reveal any changes which might account for the significantly different 5'-nucleotidase activities. The mechanism responsible for this reduced activity of UshAc has hitherto not been known. Sequence analysis of Salmonella ushA ${ }^{+}$ and ush $A^{c}$ alleles indicated that the relative inactivity of Ush $A^{c}$ may be due to one, or more, of four amino acid substitutions. One of these changes (S139Y) is in a sequence motif that is conserved in $5^{\prime}$-nucleotidases across a range of diverse prokaryotic and eukaryotic species. Site-directed mutagenesis confirmed that a Tyr substitution of Ser-139 in Salmonella UshA ${ }^{+}$was solely responsible for loss of $5^{\prime}$-nucleotidase activity. It is concluded that the corresponding single missense mutation is the cause of the UshAc phenotype. This is the first reported instance of gene inactivation in natural isolates of bacteria via a missense mutation. These results support a model of evolution of new enzymes involving a 'silent gene' which produces an inactive, or relatively inactive, product, and are also consistent with the evolution of a novel, but unknown, enzyme specificity by a single amino acid change.
\end{abstract}

Keywords: UDP-sugar hydrolase, 5'-nucleotidase, evolution, silent gene, cryptic gene

\section{INTRODUCTION}

The evolution of new or improved enzyme specificities in bacteria may occur by two major mutational

Abbreviation: ET, electrophoretic type.

The GenBank accession numbers for the sequences determined in this work are AF188721-AF188732. mechanisms, both of which are supported by studies of experimental evolution. One mechanism involves single or multiple amino acid substitutions which may, or may not, result in partial or complete loss of the original enzyme activity (Clarke, 1974; Hall, 1981; Hartley, 1984). A second mechanism involves gene duplication, followed by silencing of one of the duplicates at the transcriptional or translational level (a 'silent gene 
intermediate'). Further evolution of this 'silent gene', initially silenced by a mutation causing reduced transcription or a reduced level of functional product, which is maintained in the population for a finite period of time, then ensues via accumulation of a variety of additional mutations. Eventually such a gene may be lost by deletion but, before this occurs, it may instead acquire a functional, albeit modified, enzymic form that will persist if of selective value to the cell (Koch, 1972; Hartley, 1974, 1979, 1980, 1984; Rigby et al., 1974; Beacham, 1987). The term 'silent gene' has been previously used to include the possibility that, in one allele of the duplicates, lack of a functional gene product may also result from a missense mutation(s) causing, for example, aberrant folding of the encoded protein (Hartley, 1974; Rigby et al., 1974). The high level of gene paralogy in prokaryotic genomes indicates that gene duplication is a major mechanism for generating biochemical diversity (Labedan \& Riley, 1995; Riley \& Labedan, 1997).

Cryptic genes are also not usually expressed, i.e. are phenotypically silent at the level of transcription or translation, but do not have permanent loss of normal function. They can be periodically activated in a few members of a population by decryptification (reversion) under appropriate environmental circumstances to provide a selective advantage. Thus, cryptic genetic systems, involving cycles of inactivation and reactivation, represent a form of long-term gene regulation of rarely utilized functions operating at the level of bacterial populations (Hall et al., 1983; Hall, 1989).

Since the term 'silencing' has recently become more commonly associated with genes which are transcriptionally or translationally inactive or with partitioning sequences (Rine, 1999), use of the term 'silent gene' may imply lack of expression at the protein level. Because this is not necessarily always the case, we suggest instead that cryptic genes may, in principle, be of two types: those which encode a known function which is not normally expressed (as in the case of cryptic gene regulatory systems), and those which are expressed but whose function is unknown or whose protein product is simply inactive due to, for example, misfolding; the latter may be intermediates in the evolution of new genes (see above; Hartley, 1974; Rigby et al., 1974). Where lack of a protein product is known, the corresponding gene designation is superscript ' 0 ', as in the case of known cryptic gene regulatory systems. Where the mechanism of gene inactivation is not known, or where the expressed protein has lost its original activity but may, or may not, have acquired novel substrate specificity, we suggest a gene designation of superscript 'c'.

In the course of studies on the ush genes in Salmonella enterica and Escherichia coli, we have identified two inactive alleles, originally designated $u s h A^{0}$ and $u s h B^{0}$, respectively (Burns et al., 1983; Burns \& Beacham, 1986a, b; Edwards et al., 1993). E. coli K-12 and S. enterica serotype Typhimurium (synonym of Salmonella typhimurium) LT2 synthesize biochemically and genetically distinct UDP-sugar hydrolases, encoded by the $u s h A$ and $u s h B$ genes, respectively. The E. coli ush $A$ gene encodes a periplasmic enzyme which is bifunctional, also possessing $5^{\prime}$-nucleotidase activity. This enzyme belongs to a superfamily of phosphoesterases and displays substantial sequence homology to $5^{\prime}$ nucleotidases from a variety of sources, many of which also have UDP-sugar hydrolase activity (Zimmermann, 1992; Koonin, 1994). In contrast, the ushB-encoded UDP-sugar hydrolase from Salmonella does not possess $5^{\prime}$-nucleotidase activity, is membrane-bound, and is unrelated immunologically and in primary sequence (Garrett et al., 1989; Jones et al., 1993).

It has previously been documented that $S$. enterica serotype Typhimurium strain LT2 does not possess a 5'nucleotidase (Glaser et al., 1967; Neu, 1968; see also Burns \& Beacham, 1986a). However, when the cloned $u s h A$ gene from $E$. coli was used to probe the genomes of strain LT2 and natural isolates of S. enterica serotype Typhimurium using Southern hybridization, a homologous sequence was identified (Burns \& Beacham, 1986a). The LT2 homologue was cloned and characterized, and subsequently designated $u s h A^{0}$ (Burns \& Beacham, 1986a); in this paper, we now refer to this allele as $u s h A^{\mathrm{c}}$ since an inactive protein product is made (see Results). Further, most natural isolates of S. enterica serotype Typhimurium from a variety of sources, as well as strains of serotypes Gallinarum and Pullorum, have either no detectable, or very low, levels of $5^{\prime}$-nucleotidase activity (Neu, 1968; Burns \& Beacham, 1986a; Edwards et al., 1993) but, like strain LT2, were shown by Southern analysis to contain homologues using an ush $A^{\mathrm{c}}$-specific probe (Edwards et al., 1993; D. Innes, unpublished data). In contrast, all other natural isolates of $S$. enterica examined, representing all seven Salmonella subgroups, were found to contain active 5'nucleotidases (Edwards et al., 1993).

The cloned $u s h A$ and $u s h A^{\mathrm{c}}$ genes were shown to be located in homologous regions of their respective genomes, and to be $80 \%$ identical at the DNA sequence level and $87 \%$ identical at the amino acid level, the latter equating to 71 residue differences among the 550 residues. The $u s h A^{\mathrm{c}}$ protein was shown to be immunologically related to UshA and, like its counterpart in $E$. coli, to be located in the periplasm (Burns \& Beacham, 1986a). Examination of the LT2 ush $A^{\mathrm{c}}$ sequence did not reveal any premature stop codons, internal deletions, nor any other detrimental alterations which might account for its greatly reduced 5 -nucleotidase activity in comparison with its E. coli homologue. This is in contrast to other well-known E. coli-Salmonella differences (e.g. lac, phoA) where the lack of functional Salmonella genes is due to the absence of the corresponding chromosomal segments (Riley \& Sanderson, 1990). We have previously suggested that the ush $A^{\mathrm{c}}$ allele is not a component of a cryptic regulatory system, but rather has been very recently 'silenced', and may be destined for eventual deletion from the genome (Burns \& Beacham, 1986a; Beacham, 1987; Edwards et al., 1993). 
The mechanism of $u s h A^{\mathrm{c}}$ inactivation, however, has hitherto not been known. In this work, we provide evidence that $u s h A^{\mathrm{c}}$ alleles contain a missense mutation that results in a non-conserved amino acid substitution within a region which is generally conserved amongst similar enzymes from diverse prokaryotic and eukaryotic sources. We conclude that the corresponding single missense mutation is the cause of the $\mathrm{UshA}^{\mathrm{c}}$ phenotype. This is the first reported instance of gene inactivation in natural isolates of bacteria via a missense mutation. This work supports a model of evolution of new enzymes involving a 'silent gene' which produces an inactive, or relatively inactive, product, and is also consistent with the evolution of a novel, but unknown, enzyme specificity by a single amino acid change.

\section{METHODS}

Bacterial strains. The Salmonella strains used in this study were taken from DNA subgroup I as this is the only group that has members with ush $A^{\mathrm{c}}$ alleles (Edwards et al., 1993). All strains used are serotypes of $S$. enterica and the designations for each strain have been abbreviated to their serotype names for simplicity. Where known, the electrophoretic type (ET) for each strain is also indicated (see Edwards et al., 1993; Reeves et al., 1989). The strains used were as follows: $S$. Typhimurium LT2, S. Agona CDC 3531-75 (ET9), S. Cholerae-suis CDC 290-71 (ET11), S. Gallinarum CDC 2950-79 (ET13), S. Hadar CDC 585-86 (ET8), $S$. Heidelberg CDC 4689-70 (ET6), $S$. Heidelberg 10540 J90, S. Pullorum CDC 2385-70 (ET14), S. Typhimurium CDC 137-85 (ET5), S. Typhimurium CDC 331-86 (ET3), S. Typhimurium CDC 655-84 (ET3) and $S$. Typhimurium CDC 1119-83 (ET3). Sources of these organisms are as previously described (Edwards et al., 1993; Reeves et al., 1989). To create an E. coli chromosomal deletion of ushA, regions $5^{\prime}$ and $3^{\prime}$ were amplified using PCR and cloned into the R6K $\gamma$ oriR plasmid, pS1080 (Bass et al., 1996), creating pML1. This plasmid was then recombined into the chromosome of strain 46C5 [DE3(lac)X74 $\Delta p h o A 532$ rep-71 phn(EcoB)/ F'pOX38::Tn10-11] by M13 transduction and carbenicillin selection (Steed \& Wanner, 1993). P1 lysates were made on the resulting carbenicillin-resistant chromosomal integrates and transduced into strain W3110 (Hill \& Harnish, 1981). Carbenicillin-resistant transductants were patched onto sucrose media (Bass et al., 1996) at $25^{\circ} \mathrm{C}$ for selection of plasmid resolvants. Sucrose-resistant, carbenicillin-sensitive colonies were screened for the deleted $u s h A$ gene by DNA amplification using oligonucleotides flanking the locus. One W3110 $\Delta u s h A$ isolate was stored as strain $51 \mathrm{G} 1$.

Plasmids. All plasmids used in this study were pBluescript $\mathrm{SK}(+)$ (Stratagene) derivatives. pSM1 was generated by inserting the $u s h A^{\mathrm{c}}$-encoding $2.62 \mathrm{~kb} \mathrm{MscI}$ fragment from pDBS1 (Burns \& Beacham, 1986a) into EcoRV-cut vector. p2.4 was generated by inserting a $2 \mathrm{~kb}$ PCR-amplified fragment containing the $u s h A^{+}$gene from $S$. enterica serotype Typhimurium CDC 137-85 into KpnI/HindIII-cut vector. The amplification primer pair used corresponds to nucleotide positions 53-75 and 2026-2048 (Burns \& Beacham, 1986a), and incorporated add-on sequences to generate these unique restriction sites in the amplified product.

PCR and DNA sequencing. The $u s h A^{+}$and $u s h A^{c}$ alleles were amplified from the Salmonella strains using oligonucleotides designed to anneal 90 bases upstream of the sequence encoding the initiating methionine codon and 207 nucleotides downstream of the sequence for the $u s h A^{\mathrm{c}}$ stop codon (Burns \&
Beacham, 1986a). Amplification reactions $(50 \mu \mathrm{l})$ consisted of $1.5 \mathrm{mmol} \mathrm{MgCl}_{2}, 50 \mathrm{pmol}$ of each primer, $12.5 \mu \mathrm{mol} \mathrm{dNTP}$ mix ( $25 \mathrm{mM}$ each of dGTP, dATP, dTTP, dCTP) and $5 \mu \mathrm{l}$ of a suspension of a single colony in $100 \mu \mathrm{l}$ distilled water. Reactions were placed in a thermal cycler (preheated to $95^{\circ} \mathrm{C}$ ) for $7 \mathrm{~min}$, prior to the addition of 2 units of Taq polymerase (Promega). The thermal cycling programme comprised 35 cycles of $95^{\circ} \mathrm{C}$ for $30 \mathrm{~s}, 50{ }^{\circ} \mathrm{C}$ for $90 \mathrm{~s}$ and $72{ }^{\circ} \mathrm{C}$ for $60 \mathrm{~s}$. PCR products were purified from $0.7 \%$ agarose gels using QiaEX II resin (Qiagen) and directly sequenced using the Prism Dye Terminator Cycle Sequencing reagents (Applied Biosystems). Sequencing primers were designed to anneal to the following nucleotide positions of the $u s h A^{\mathrm{c}}$ coding strand (Burns \& Beacham, 1986a): 319-333, 571-585, 835-849, 1091-1105, 1279-1299, 1484-1498 and 1691-1709. A similar set of primers was designed for determining the complementary sequence. These primers annealed to the following positions within the ush $A^{\mathrm{c}}$ sequence: $307-321,546-560,796-810,1048-1062$ and 1301-1315. Sequencing reactions were electrophoresed on an Applied Biosystems 373A automated sequencer.

Immunoblotting. Immunoblots were performed as described by Ausubel et al. (1987). Protein samples were transferred to PVDF membrane using a Bio-Rad Transblotting cell. The primary antibody was a polyclonal UshA antibody from rabbit serum (Beacham \& Wilson, 1982). Bands were detected with a goat anti-rabbit-horse radish peroxidase conjugate and 3',3'-diaminobenzidine/ $\mathrm{NiCl}_{2}$ colour development solution.

Site-directed mutagenesis. The mutants described in this study were generated using the methods of Eckstein et al. (Taylor et al., 1985; Nakamaye \& Eckstein, 1986) and Kunkel (1985), using reagents supplied by Amersham and Bio-Rad, respectively. The change of Phe-463 (nt positions 1552-1554) to a Leu residue was introduced using a 39-mer annealing to the region corresponding to nt positions 1535-1573 of $u s h A^{\mathrm{c}}$, and the change of Ser-139 (nt positions 580-582) to a Tyr residue was introduced using a 21 -mer annealing to the region corresponding to nt positions 571-591 of $u s h A^{\mathrm{c}}$ (see Burns \& Beacham, 1986a).

Enzyme assay. Assays of $5^{\prime}$-nucleotidase were performed as previously described using periplasmic extracts (Edwards et al., 1993). For each culture, an equal amount of periplasmic extract was added into three reaction mixes; each of these triplicate assays was sampled at four time points during the reaction providing a mean activity with less than $3 \%$ variance. A comparison of the measured activities of at least three independently grown cultures of each isolate revealed a similarly low level of variance. Periplasmic extracts were prepared from $40 \mathrm{ml}$ R-broth cultures grown aerobically at $37^{\circ} \mathrm{C}$ to $\mathrm{OD}_{450} 0 \cdot 8$; R-broth contains (per litre) $10 \mathrm{~g}$ tryptone, $5 \mathrm{~g} \mathrm{NaCl}, 1 \mathrm{~g}$ yeast extract, $1 \mathrm{~g}$ glucose. The cells were harvested by centrifugation and the cells resuspended in $1.8 \mathrm{ml}$ of a solution containing $15 \%$ sucrose, $50 \mathrm{mM}$ Tris $/ \mathrm{HCl}$, pH 8.0. Lysozyme (140 $\mu \mathrm{l}$ of a $2 \mathrm{mg} \mathrm{ml}^{-1}$ stock) and $40 \mu \mathrm{l} 0 \cdot 1 \mathrm{M}$ EDTA were added and the mixture was incubated at $25^{\circ} \mathrm{C}$ for $10 \mathrm{~min}$. Spheroplasts were removed by centrifugation at 8000 r.p.m. (Sorvall SS34 rotor) and the supernatant was carefully transferred to a fresh tube. The centrifugation step was repeated and supernatants (periplasmic fractions) were aliquoted into fresh tubes and assayed immediately, or stored at $-80{ }^{\circ} \mathrm{C}$ until required. Specific activity was expressed as units $(\mathrm{mg} \text { protein })^{-1}$, where a unit is defined as nmol $\mathrm{P}_{\mathrm{i}} \mathrm{min}^{-1}$. Assays of UDP-sugar hydrolase were also performed as previously described (Edwards et al., 1993) except that activities were measured in periplasmic extracts. Specific activity was expressed as units (mg protein $)^{-1}$, where a unit is defined as ( $\mu$ mol UDP-glucose hydrolysed) $\mathrm{min}^{-1}$. 
Sequence analysis. 5'-Nucleotidase sequences were identified following a BLAST search (Altschul et al., 1990) of GenBank, PDB, SWISS-PROT, PIR and PRF databases. Fully annotated sequences resulting from the search were extracted and edited, leaving only the mature portions of each primary sequence. These sequences were aligned using CLUSTAL $\mathrm{x}$ (Thompson et al., 1994) and analysed using Prosite (Bairoch, 1992) and Blocks (Henikoff \& Henikoff, 1992) databases.

\section{RESULTS}

\section{Enzymic analysis of ushA and ushAc strains of Salmonella}

We have previously shown that $u s h A^{c}$ alleles show mainly a clonal distribution amongst a set of isolates representing the seven DNA subgroups of Salmonella, whose genetic relationship was determined from multilocus enzyme electrophoresis (Reeves et al., 1989). Accordingly, for this study, a set of ush $A$ and $u s h A^{\mathrm{C}}$ strains representing DNA subgroup I of Salmonella (see Reeves et al., 1989; Edwards et al., 1993) was chosen for analysis. The specific activities of $5^{\prime}$-nucleotidase in these strains are shown in Table 1, together with their allocated phenotypes. It was found that three strains (CDCs 1119-83, 655-84, 331-86) of one particular electrophoretic type, ET3, are UshA ${ }^{\mathrm{c}}$ and these are all of serotype Typhimurium (Edwards et al., 1993). Only one serotype Typhimurium strain (CDC 137-85) has normal $\mathrm{UshA}^{+}$specific activity, but, significantly, this is a relatively unrelated strain (Edwards et al., 1993). The only other UshA ${ }^{\mathrm{c}}$ strains of S. enterica (CDCs 2950-79 and 2385-70) are of serotypes Gallinarum (ET13) and Pullorum (ET14), respectively.

UDP-sugar hydrolase activities of ush $A$ - and $u s h A^{\mathrm{c}}$. encoded periplasmic proteins could not be readily determined in these natural Salmonella isolates since

Table 1. $5^{\prime}$-Nucleotidase activity in ush $A^{c}$ and $u s h A^{+}$ strains of Salmonella

\begin{tabular}{|lcrc|}
\hline Strain & $\begin{array}{c}\text { ush } \boldsymbol{A}^{+/} \\
\text {ush } \boldsymbol{A}^{\mathrm{c}}\end{array}$ & ET $^{*}$ & $\begin{array}{c}\text { Specific } \\
\text { activity }\end{array}$ \\
\hline S. Heidelberg CDC 4689-70 & ush $A^{+}$ & 6 & $36 \cdot 6$ \\
S. Heidelberg 10540 J90 & ush $A^{+}$ & - & $32 \cdot 1$ \\
S. Cholerae-suis CDC 290-71 & ush $A^{+}$ & 11 & $19 \cdot 4$ \\
S. Agona CDC 3531-75 & ush $A^{+}$ & 9 & $26 \cdot 7$ \\
S. Hadar CDC 585-86 & ush $A^{+}$ & 8 & $26 \cdot 2$ \\
S. Typhimurium CDC 137-85 & ush $A^{+}$ & 5 & $31 \cdot 1$ \\
S. Typhimurium LT2 & ush $A^{\mathrm{c}}$ & - & $0 \cdot 39$ \\
S. Typhimurium CDC 1119-83 & ush $A^{\mathrm{c}}$ & 3 & $0 \cdot 68$ \\
S. Typhimurium CDC 331-86 & ush $A^{\mathrm{c}}$ & 3 & $0 \cdot 22$ \\
S. Typhimurium CDC 655-84 & ush $A^{\mathrm{c}}$ & 3 & $0 \cdot 39$ \\
S. Gallinarum CDC 2950-79 & ush $A^{\mathrm{c}}$ & 13 & $0 \cdot 43$ \\
S. Pullorum CDC 2385-70 & ush $A^{\mathrm{c}}$ & 14 & $0 \cdot 17$ \\
\hline
\end{tabular}

*ET, electrophoretic type (see Edwards et al., 1993; Reeves et al., 1989). -, Unknown.

$\dagger$ Specific activity is expressed as nmol $\mathrm{P}_{\mathrm{i}} \min ^{-1} \mathrm{mg}^{-1}$. they also contain an active membrane-bound UDPsugar hydrolase encoded by $u s h B^{+}$alleles. To address this complication, plasmid-encoded $u s h A^{+}$and $u s h A^{\mathrm{c}}$ genes from CDC 137-85 and LT2, respectively, were transformed into a $\Delta u s h A E$. coli $51 G 1$ strain. Like other $E$. coli strains, this strain lacks an active $u s h B$-encoded UDP-sugar hydrolase. Specific activities of UDP-sugar hydrolase from cloned Salmonella ush $A^{+}$and $u s h A^{c}$ alleles in these transformants were found to correlate with those for chromosomally encoded $5^{\prime}$-nucleotidase in corresponding Salmonella isolates. The former was found to have a specific UDP-sugar hydrolase activity of 1.22 units $\mathrm{mg}^{-1}$ whereas the latter had undetectable activity $\left(<2.75 \times 10^{-3}\right.$ units $\left.\mathrm{mg}^{-1}\right)$. Irrespective of which of the two enzymic activities are considered, the allocated phenotypes as $\mathrm{UshA}^{+}$or $\mathrm{UshA}^{\mathrm{c}}$ are consistent.

\section{Immunoblot analysis of ushA and ushA' strains of Salmonella}

To determine whether the $u s h A^{c}$ allele is expressed at the translational level in natural $S$. enterica isolates, the presence of the protein in cell extracts was examined using immunoblotting. The results clearly indicated the presence of a cross-reactive polypeptide of molecular mass indistinguishable from native $5^{\prime}$-nucleotidase (Fig. $1)$. The presence of a second cross-reactive polypeptide of higher molecular mass ( $\sim 85 \mathrm{kDa}$; see Fig. 1) has been previously observed (Burns \& Beacham, 1986a) but its identity is not known. From this experiment we conclude that the $u s h A^{\mathrm{c}}$ allele is transcribed and translated, producing a mature protein.

\section{Comparative nucleotide sequence analysis of ushA and $u s h A^{c}$ alleles}

To investigate the mechanism of inactivation of $\mathrm{UshA}^{\mathrm{c}}$, we have sequenced the $u s h A^{+}$and $u s h A^{c}$ alleles from all of the Salmonella strains listed in Table 1. The 5'untranslated sequences, up to 62 nt from the initiation codon, are invariant amongst these strains (data not shown) and are the same as the $u s h A^{\mathrm{c}}$ allele from strain LT2 (see Burns \& Beacham, 1986a). They include the Shine-Dalgarno region, which is the same as in the $u s h A^{+}$gene of $E$. coli, and the -10 and -35 regions, identified on the basis of transcription mapping of the $u s h A^{+}$allele from E. coli (Burns \& Beacham, 1986b). There is an identical single nucleotide change in their -10 regions when compared with E. coli (Burns \& Beacham, 1986a). All of these observations are in accord with the fact that the $u s h A^{\mathrm{c}}$ gene encodes a protein product.

We hence considered it likely that an amino acid change(s) in the UshA polypeptide results in a relatively inactive $5^{\prime}$-nucleotidase (see Table 1 ). Two approaches were used to identify those amino acid residues which merited further investigation.

First, the coding sequences of the Salmonella $u s h A^{+}$and $u s h A^{c}$ genes were examined for nucleotide substitutions that occurred in at least three of the sequences at any 


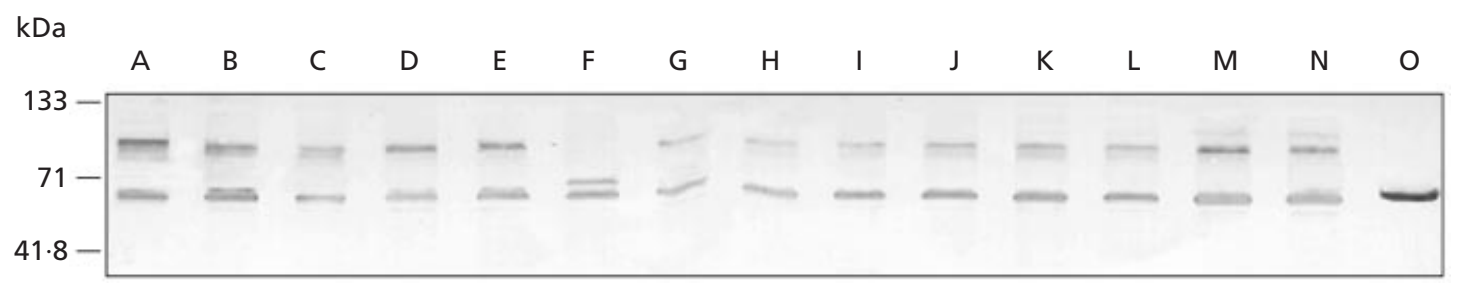

Fig. 1. Immunoblot of UshA and UshA ${ }^{c}$ proteins in cell extracts of Salmonella strains. Molecular mass markers are indicated to the left of the panel (kDa). Lanes: A, E. coli; B, S. Heidelberg CDC 4689-70; C, S. Heidelberg 10540 J90; D, S. Agona CDC 3531-75; E, S. Hadar CDC 585-86; F, S. Cholerae-suis CDC 290-71; G, S. Typhimurium CDC 137-85; H, S. Typhimurium CDC 179-87; I, S. Typhimurium LT2; J, S. Typhimurium CDC 1119-83; K, S. Typhimurium CDC 655-84; L, S. Typhimurium CDC 331-86; M, S. Gallinarum CDC 2950-79; N, S. Pullorum CDC 2385-70; O, purified Ush (200 ng).

Table 2. Amino acid substitutions in ush $A^{c}$ and $u s h A^{+}$strains of Salmonella

\begin{tabular}{|c|c|c|c|c|c|c|c|}
\hline \multirow[t]{2}{*}{ Strain } & \multirow[t]{2}{*}{$u \operatorname{sh} A^{+} / u \operatorname{sh} A^{\mathrm{c}}$} & \multirow[t]{2}{*}{$\mathrm{ET}^{*}$} & \multicolumn{5}{|c|}{ Amino acid codon: $†$} \\
\hline & & & 139 & 347 & 449 & 463 & 544 \\
\hline S. Heidelberg CDC 4689-70 & $u s h A^{+}$ & 6 & Ser & Ser & Ala & Phe & Asn \\
\hline S. Heidelberg $10540 \mathrm{~J} 90$ & $u s h A^{+}$ & - & Ser & Ser & Ala & Phe & Asn \\
\hline S. Cholerae-suis CDC 290-71 & $u s h A^{+}$ & 11 & Ser & Ser & Ala & Phe & Asn \\
\hline S. Agona CDC 3531-75 & $u s h A^{+}$ & 9 & Ser & Ser & Ala & Phe & Lys \\
\hline S. Hadar CDC 585-86 & $u s h A^{+}$ & 8 & Ser & Ser & Ala & Phe & Lys \\
\hline S. Typhimurium CDC 137-85 & $u s h A^{+}$ & 5 & Ser & Ser & Ala & Phe & Lys \\
\hline S. Typhimurium LT2 & $u s h A^{\mathrm{c}}$ & - & Tyr & Ser & Ala & Leu & Asn \\
\hline S. Typhimurium CDC 1119-83 & $u s h A^{\mathrm{c}}$ & 3 & Tyr & Ser & Ala & Leu & Asn \\
\hline S. Typhimurium CDC 331-86 & $u s h A^{\mathrm{c}}$ & 3 & Tyr & Ser & $\operatorname{Arg}$ & Leu & Asn \\
\hline S. Typhimurium CDC 655-84 & $u s h A^{\mathrm{c}}$ & 3 & Tyr & Ser & $\operatorname{Arg}$ & Leu & Asn \\
\hline S. Gallinarum CDC 2950-79 & $u s h A^{\mathrm{c}}$ & 13 & Ser & Leu & $\operatorname{Arg}$ & Leu & Lys \\
\hline S. Pullorum CDC 2385-70 & $u s h A^{\mathrm{c}}$ & 14 & Ser & Leu & $\operatorname{Arg}$ & Leu & Lys \\
\hline
\end{tabular}

*ET, electrophoretic type (see Edwards et al., 1993; Reeves et al., 1989). -, Unknown.

† The nucleotide positions (see Burns \& Beacham, 1986a) corresponding to these codons are: 580-582 (codon 139), 1204-1206 (codon 347), 1510-1512 (codon 449), 1552-1554 (codon 463) and 1795-1797 (codon 544).

given nucleotide position, which would potentially reveal amino acid substitutions for which a possible correlation to phenotype could be established. Through such an analysis, we discovered 13 nucleotide differences, of which 8 occur at the $3^{\prime}$ base of codons (data not shown) and therefore do not result in amino acid substitutions. The remaining five nucleotide differences were examined to see whether they result in conservative or non-conservative amino acid substitutions, and for correlation to phenotype. These nucleotide changes have led to four amino acid substitutions, of which two are conservative (at positions 463 and 544; Table 2) and two non-conservative (at positions 139 and 449; Table 2). We conclude that one or more of these alterations are responsible for the loss of $5^{\prime}$-nucleotidase activity of the $u s h A^{\mathrm{c}}$ gene product.

The second approach involved ordered pairwise comparisons of the UshA and UshA ${ }^{\mathrm{c}}$ amino acid sequences to identify all residue differences. Depending on the two sequences examined, the numbers of substitutions ranged from zero to seven residues (data not shown). All amino acid changes were then analysed for possible correlation to phenotype. In addition to the four residues already identified using the first approach, another candidate residue (at position 347; Table 2) was identified. However, this residue is of possible interest only in relation to the Ush ${ }^{c}$ phenotype of serotypes Gallinarum and Pullorum.

\section{Comparative amino acid sequence analysis of 5'-nucleotidases from diverse sources}

Notwithstanding the availability of the threedimensional structure of E. coli 5'-nucleotidase (Knöfel \& Sträter, 1999), we sought to gain insight into which of these residues might be important for the catalytic function of UshA. This was done by aligning amino acid sequences of 5'-nucleotidases from diverse sources 


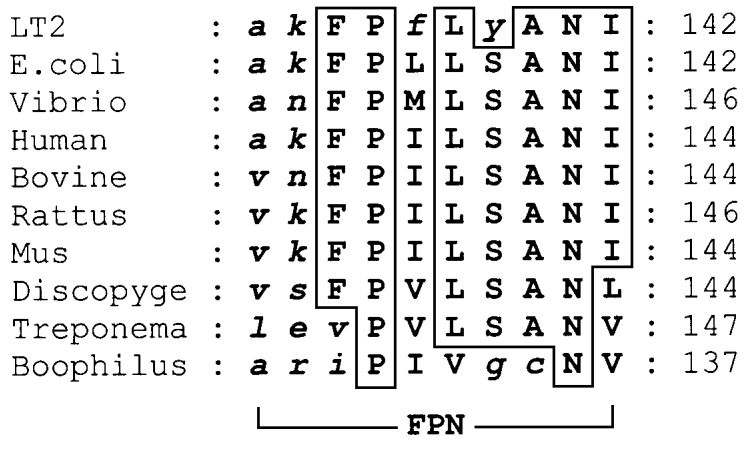

Fig. 2. Alignment of mature $5^{\prime}$-nucleotidase sequences. The one-letter code for amino acids is used. The sequence of the $S$. enterica serotype Typhimurium strain LT2 $5^{\prime}$-nucleotidase is shown on the top line. The other sequences are of active $5^{\prime}$ nucleotidases from various sources: E.coli, E. coli K-12 (Burns \& Beacham, 1986b); Vibrio, Vibrio parahaemolyticus (Tamao et al., 1991); Human, Homo sapiens (Misumi et al., 1990a); Bovine, Bos taurus (Suzuki et al., 1993); Rattus, Rattus norvegicus (Misumi et al., 1990b); Mus, Mus musculus (Resta et al., 1993); Discopyge, Discopyge ommata (Volknandt et al., 1991); Treponema, Treponema pallidum (Fraser et al., 1998); Boophilus, Boophilus microplus (Liyou et al., 1999). Numbers indicate the position of each residue at the end of the FPN motifs shown, with respect to the sequences of corresponding precursor proteins. The ' $y$ ' residue on the top line is Tyr-139 (see text). Boxed residues show sequence identity in at least 7 of the 10 sequences shown; upper-case unboxed residues show 'strong' sequence similarity while the lower-case italicized residues show 'weaker' similarity, as determined by weights assigned in the BLOSUM62 matrix (Henikoff \& Henikoff, 1992) and the CLUSTAL program (Thompson et al., 1994).

together with the amino acid sequence derived from the $u s h A^{c}$ allele from Salmonella strain LT2. This examination resulted in the identification of a number of individual residues which are conserved across all of the enzymes. In addition, a number of signature motifs of three or more residues which display sequence identity across all sequences were also identified (data not shown). Of the five residues listed in Table 2, only Ser139 is conserved in diverse active $5^{\prime}$-nucleotidases and is contained within the 'FPN' motif. The FPN sequence of UshA $^{c}$ in Salmonella strain LT2 contains a nonconservative Ser to Tyr substitution (Fig. 2). Tyr-139 is also found in all other serotype Typhimurium UshA ${ }^{\mathrm{c}}$ strains examined (Table 2). We therefore predicted this substitution to be responsible for the loss of $5^{\prime}$ nucleotidase activity of $\mathrm{UshA}^{\mathrm{c}}$ in serotype Typhimurium. However, in common with all examined UshA ${ }^{+}$ strains, strains of serotypes Gallinarum and Pullorum have a Ser-139 residue, indicating that an independent mutation(s) is responsible for their $\mathrm{UshA}^{\mathrm{c}}$ phenotype (see Discussion).

\section{Site-directed mutagenesis of the ushA ${ }^{+}$gene}

To substantiate the hypothesis that the Ser to Tyr substitution abolishes $5^{\prime}$-nucleotidase activity in most strains of serotype Typhimurium, we have reproduced
Table 3. 5'-Nucleotidase activity in site-directed mutants of ush $A^{c}$

Three individual mutant isolates from each mutagenesis were assayed using periplasmic extracts. Assays on whole cells were also performed with identical relative values (data not shown).

\begin{tabular}{|lccc|}
\hline Plasmid* & \multicolumn{2}{c|}{ Amino acid codon: } & Specific activity $\dagger$ \\
\cline { 2 - 3 } & $\mathbf{1 3 9}$ & $\mathbf{4 6 3}$ & \\
\hline None & - & - & $<1.0$ \\
p2.4 & Ser & Phe & 893 \\
p1387C-T & Ser & Leu & $1.7 \times 10^{3}$ \\
pSM1 & Tyr & Leu & 8.7 \\
pCA-D & Tyr & Phe & 11.7 \\
\hline
\end{tabular}

*All strains are $51 \mathrm{G} 1(\Delta u s h A)$ containing the indicated plasmid, or no plasmid. Plasmid p2.4 contains the $u s h A^{+}$gene from $S$. Typhimurium CDC 137-85; pSM1 contains $u s h A^{\mathrm{c}}$ from $S$. Typhimurium LT2; p1387C-T and pCA-D are site-directed mutants derived from $\mathrm{p} 2.4$, as indicated.

† Specific activity is expressed as $\mathrm{nmol} \mathrm{P}_{\mathrm{i}} \mathrm{min}^{-1} \mathrm{mg}^{-1}$.

this change by creating a $\mathrm{C} \rightarrow \mathrm{A}$ substitution at the second position of codon 139 in the active $u s h A^{+}$gene from $S$. enterica serotype Typhimurium strain CDC 137-85 (see Methods). Likewise, we altered residue 463 from Phe to Leu, which is associated with the UshA ${ }^{\mathrm{c}}$ phenotype (see Table 2), inclusive of the strains with serotypes Gallinarum and Pullorum. Each mutated gene was introduced into an $\Delta u s h A$ genetic background and assayed for $5^{\prime}$-nucleotidase activity.

The results (Table 3) showed that the substitution of Phe to Leu at residue 463 did not reduce the level of $5^{\prime}$ nucleotidase activity, and in fact increased it. However, the Ser to Tyr change at residue 139 abolished 5'nucleotidase activity. Thus, we had selectively and specifically reproduced the $U_{s h A}{ }^{c}$ phenotype and we concluded that a $\mathrm{C} \rightarrow \mathrm{A}$ substitution within codon 139 of $u s h A^{\mathrm{c}}$ is the cause of inactivation of $5^{\prime}$-nucleotidase in most $S$. enterica serotype Typhimurium isolates.

\section{DISCUSSION}

\section{ush $A^{c}$ is inactivated in most strains of serotype Typhimurium by a single missense mutation}

We have previously shown that the origin of the inactivating mutation leading to loss of $5^{\prime}$-nucleotidase activity appears to be clonal (Edwards et al., 1993): isolates of one Typhimurium ET (ET3) are all UshA ${ }^{\mathrm{c}}$. Only a single, relatively unrelated, Typhimurium strain (CDC 137-85) of different ET (ET5) is UshA ${ }^{+}$.

In this study, we have shown that the Ush ${ }^{\mathrm{c}}$ phenotype is due to a missense mutation in one codon, at position 139 , resulting in a non-conservative amino acid substitution (S139Y) within a highly conserved peptide 


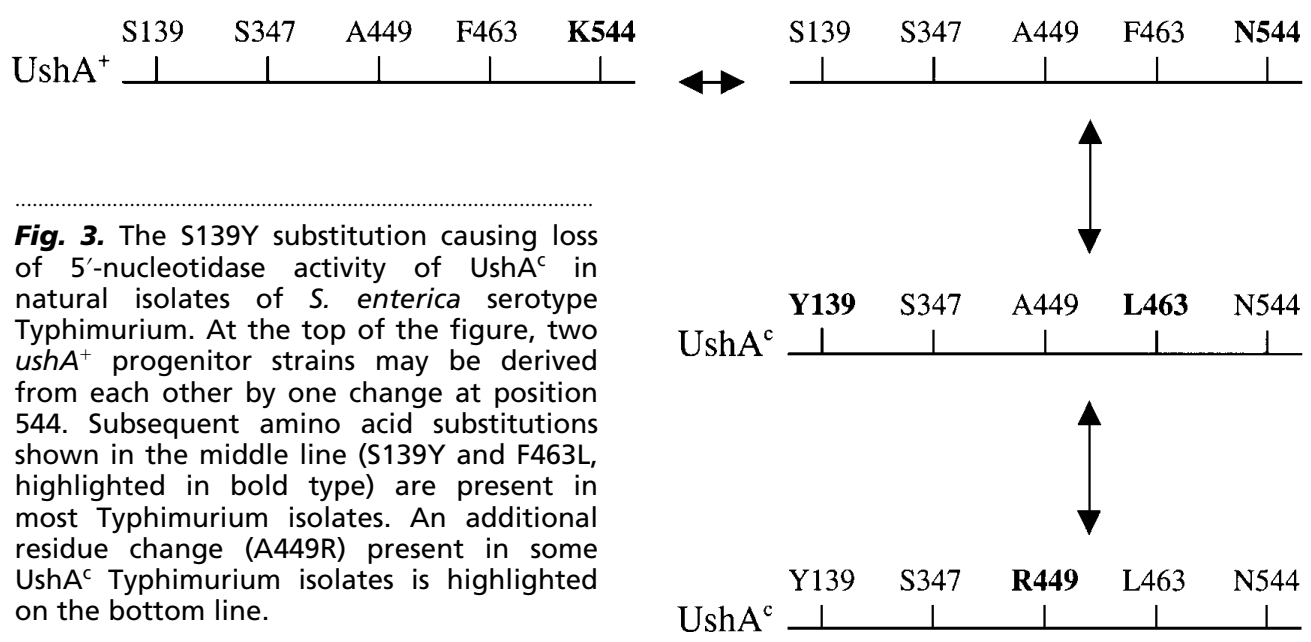

(FPN) motif. This amino acid substitution was due to a single $\mathrm{C} \rightarrow \mathrm{A}$ nucleotide change. In the threedimensional structure of E. coli UshA (Knöfel \& Sträter, 1999), this residue is located between $\beta$-sheets 4 and 5 and does not appear to be within the active site. Computer simulation of this substitution predicts no significant effect on tertiary structure (data not shown). Nevertheless, the location of this residue within the conserved FPN motif suggests an important role in structure and/or function.

Fig. 3 summarizes the amino acid substitution data presented in Table 2 in the context of depicting how the postulated mutational change (see below) has led to the inactivation of the $u s h A^{\mathrm{c}}$ gene in natural isolates of $S$. enterica serotype Typhimurium. The independent mutation(s) responsible for the inactivation of the $u s h A^{c}$ alleles in serotypes Gallinarum and Pullorum are not included in this figure but are discussed below.

This study is the first report of gene inactivation by missense mutation occurring in natural isolates of bacteria, leading to an expressed yet relatively inactive (5'-nucleotidase) enzyme. This is quite distinct from gene inactivation (or deletion) as a consequence of laboratory cultivation in rich medium, and confirms the importance of examining natural isolates (Delorme et al., 1993; Burns et al., 1995).

\section{ushAc in other Salmonella serotypes}

All other Salmonella isolates examined are $\mathrm{UshA}^{+}$except for two additional strains, one Gallinarum and one Pullorum (ET13 and ET14, respectively), which were found to be phenotypically UshA ${ }^{\mathrm{c}}$ (Edwards et al., 1993; Table 1). As in the case of CDC 137-85 (serotype Typhimurium, ET5, $\mathrm{UshA}^{+}$), these strains are also relatively distantly related to those of ET3 and do not share the inactivating mutation in codon 139 . Hence, their $U_{s h A^{c}}$ phenotype is consistent with independent mutational event(s) and with the clonal origin of the $u s h A^{c}$ Typhimurium strains. The only consistent amino acid residue differences between all $\mathrm{UshA}^{+}$proteins examined and these two $\mathrm{UshA}^{\mathrm{c}}$ sequences were identified as S347L, A449R and F463L (Table 2). Of these, only A449R is a non-conservative substitution. However, the identity of the inactivating mutations responsible for the UshA ${ }^{c}$ phenotype in the Gallinarum and Pullorum strains remain to be determined.

\section{The origin of new genes}

Aside from past or present combinatorial mechanisms, there are two mutational mechanisms whereby, in principle, new or improved enzyme specificities may arise. First, there is a finite probability that 'beneficial' mutations may be acquired, resulting in one or more amino acid substitutions which do not significantly alter expression but change the properties of the resulting polypeptide. Such changes will be 'fixed' if of selective value; this may occur with partial or complete loss of the original enzymic activity. There is substantial evidence for this mechanism from studies of experimental evolution (Clarke, 1974; Hall, 1981; Hartley, 1984). In the context of $u s h A^{\mathrm{c}}$, it is possible that a new, unknown, enzymic specificity has arisen concomitant with loss of $5^{\prime}$-nucleotidase (UDP-sugar hydrolase) activity.

Second, the analysis of bacterial genomes clearly indicates that the evolution of biochemical diversity has involved substantial levels of gene duplication; at least $37 \%$ of E. coli genes, for example, have paralogous counterparts in the genome (Labedan \& Riley, 1995; Riley \& Labedan, 1997). It has previously been proposed that enzyme evolution is more efficient if one duplicate is rendered non-functional at the level of transcription or translation, later to revert to an active allele (Koch, 1972). Similarly, it has been suggested that the evolution of an enzyme family may involve " mutation of one copy to a 'silent gene' that produces an inactive product that cannot fold correctly" (Rigby et al., 1974); following genetic divergence, reversion, at the original or at another site, may restore the ability of the protein to fold correctly (Hartley, 1974; Rigby et al., 1974). If they do not revert to a new form, such 'silent genes' will inevitably be eventually deleted from the genome; their 
presence in extant genomes is therefore likely to be rare, as recent analyses of sequenced bacterial genomes confirms (Fraser et al., 2000), and is in contrast to higher eukaryotic genomes, in which the presence of pseudogenes is common (Mighell et al., 2000). Whether or not inactive alleles are retained in the genomes of prokaryotes is of significance with respect to this model for the evolution of new or improved enzyme specificities in bacteria. Our observations with the $u s h A / u s h A^{\mathrm{c}}$ system are consistent with the hypothesized 'silent gene intermediate', although the $u s h A^{\mathrm{c}}$ gene does not appear to be a paralogue of an active $u s h A$ gene. However, the unrelated $u s h B$ gene encoding an active UDP-sugar hydrolase, and present in all Salmonella strains examined (see Edwards et al., 1993), is a functional duplicate of $u s h A$ and provides a possible rationale for the inactivation of $u s h A^{\mathrm{c}}$. If the resident ' $u s h A$ ' gene in Salmonella became functionally a duplicate of $u s h B$, which had been acquired more recently, then the former would be expected to have become inactivated $\left(u s h A^{\mathrm{c}}\right)$ if the acquired gene $(u s h B)$ has a similar role and a greater selective value. UshB is a more active UDP-sugar hydrolase than both the Salmonella UshA ${ }^{\mathrm{c}}$ enzyme (see Results) and the E. coli UshA enzyme (based on cellular specific activities and measured $K_{\mathrm{m}}$ values; Glaser $e t$ al., 1967). UshB also has broader specificity for nucleosidediphosphate sugars than the E. coli UshA enzyme (Glaser et al., 1967). The fact that $u s h A^{\mathrm{c}}$ is not a tandem duplication of $u s h B$ may account for the persistence of $u s h A^{\mathrm{c}}$ in the Salmonella genome since homologous recombination between them would not occur.

Despite the lack of evidence for gene duplication preceding inactivation of $u s h A^{\mathrm{c}}$, we suggest that the existence of $u s h A^{\mathrm{c}}$ also supports the notion of 'silent gene intermediates' in the evolution of new enzyme activities.

\section{More 'cryptic' genes in sequenced genomes?}

There are ORFs present in sequenced genomes to which a function has been assigned, on the basis of sequence similarity to known genes, but for which the corresponding enzyme activity is not apparent. These ORFs have been suggested to be possibly 'remnant' genes, which have not yet been eliminated from the genome (Pollack, 1997; Cordwell, 1999). If they encode a protein product, their original enzymic activity may have been lost by virtue of missense mutation(s), as in the case of $u s h A^{\mathrm{c}}$. Alternatively, these genes may code for proteins which have unknown functions, despite the sequence similarity of their putative encoded proteins to those of known function (Pollack, 1997; Cordwell, 1999).

\section{Is ushA' a 'remnant' gene?}

Cryptic genes have been previously defined as those which are postulated to have a role in long-term gene regulation. They are normally not expressed but undergo cycles of inactivation and activation involving periodic selection, which may allow expression of the gene (i.e. reversion via single mutational events) under selective conditions (Hall et al., 1983; Hall, 1989). The question arises as to whether the $u s h A^{\mathrm{c}}$ gene should be interpreted in this way. Clearly, the single $\mathrm{C} \rightarrow \mathrm{A}$ substitution at nt position 581, responsible for $u s h A^{\mathrm{c}}$ inactivation (loss of $5^{\prime}$-nucleotidase activity) in Typhimurium isolates, may be presumed to be potentially revertible, although this has not yet been observed (Burns \& Beacham, 1986a). In cryptic gene regulatory systems, however, the great majority of natural isolates contain the cryptic (non-expressed) allele (Hall \& Betts, 1987; Burns et al., 1995). In contrast, the great majority of $S$. enterica isolates are $u s h A^{+}$ (Edwards et al., 1993). Those relatively few isolates which are $u s h A^{\mathrm{c}}$ can simply be explained by a recent $\mathrm{C} \rightarrow \mathrm{A}$ change which occurred in an ancestor of most strains of serotype Typhimurium (see Edwards et al., 1993). In summary, there is no compelling evidence that $u s h A^{\mathrm{C}}$ is part of a cryptic gene regulatory system.

Instead, ush $A^{\mathrm{c}}$ may exemplify a second category of cryptic gene, expressed at the protein level. The E. coli serotype O157:H7 uidA gene may be another such example (Feng \& Lampel, 1994). Such genes may have evolved new (unknown) specificities.

The possible acquisition of altered enzyme specificity for the $u s h A^{\mathrm{c}}$ gene product is difficult to exclude experimentally in the absence of any notion of which substrates might be tested. Alternatively, such a cryptic gene may represent a 'remnant' gene which has become redundant. This, in turn, may be due to the presence of a more active UDP-sugar hydrolase encoded by $u s h B$ (see above). It is also possible that loss of its $5^{\prime}$ nucleotidase activity may have adaptive value in that it may possibly be related to the facultative intracellular existence of Salmonella. A role for the enzyme in affecting host cell function has been suggested in the case of invasive strains of $E$. coli due to the inhibition of host protein kinases (Berger et al., 1996). Although overexpression of $5^{\prime}$-nucleotidase increases intracellular survival in vitro, it is possible that the alteration of intracellular function is maladaptive. In either case, the $u s h A^{\mathrm{c}}$ gene may have accumulated neutral or deleterious mutations and may eventually be deleted from the genome as a non-essential or maladaptive gene.

\section{ACKNOWLEDGEMENTS}

D. Innes gratefully acknowledges the receipt of an Australian Postgraduate Research Award.

\section{REFERENCES}

Altschul, S. F., Gish, W., Miller, W., Myers, E. W. \& Lipman, D. J. (1990). Basic local alignment search tool. J Mol Biol 215, 403-410.

Ausubel, F. M., Brent, R., Kingston, R. E., Moore, D. D., Seidman, J. G., Smith, J. A. \& Struhl, K. (1987). Current Protocols in Molecular Biology. New York: Greene Publishing Associates/ Wiley Interscience.

Bairoch, A. (1992). PROSITE: a dictionary of sites and patterns in proteins. Nucleic Acids Res 20, 2013-2018. 
Bass, S., Gu, Q. \& Christen, A. (1996). Multicopy suppressors of Prc mutant Escherichia coli include two HtrA (DegP) protease homologs (HhoAB), DskA, and a trucated RlpA. J Bacteriol 178, 1154-1161.

Beacham, I. R. (1987). Silent genes in prokaryotes. FEMS Microbiol Rev 46, 409-417.

Beacham, I. R. \& Wilson, M. S. (1982). Studies on the UDP-sugar hydrolases from Escherichia coli and Salmonella typhimurium. Arch Biochem Biophys 218, 603-608.

Berger, S. A., Rowan, K., Morrison, H. D. \& Ziltener, H. J. (1996). Identification of a bacterial inhibitor of protein kinases. Mechanism and role in host cell invasion. J Biol Chem 271, 23431-23437.

Burns, D. M. \& Beacham, I. R. (1986a). Identification and sequence analysis of a silent gene $\left(u s h \mathrm{~A}^{0}\right)$ in Salmonella typhimurium. J Mol Biol 192, 163-175.

Burns, D. M. \& Beacham, I. R. (1986b). Nucleotide sequence and transcriptional analysis of the E. coli ushA gene, encoding periplasmic UDP-sugar hydrolase (5'-nucleotidase) : regulation of the $u s h \mathrm{~A}$ gene, and the signal sequence of its encoded protein product. Nucleic Acids Res 14, 4325-4342.

Burns, D. M., Abraham, L. J. \& Beacham, I. R. (1983). Characterisation of the ush gene of Escherichia coli and its protein products. Gene 25, 343-353.

Burns, D. M., Burger, M. J. \& Beacham, I. R. (1995). Silent genes in bacteria: the previously designated 'cryptic' ilvHI locus of 'Salmonella typhimurium LT2' is active in natural isolates. FEMS Microbiol Lett 131, 167-172.

Clarke, P. H. (1974). The evolution of enzymes for the utilisation of novel substrates. In Evolution in the Microbial World, pp. 183-217. Edited by M. J. Carlile \& J. J. Skehel. Cambridge: Cambridge University Press.

Cordwell, S. J. (1999). Microbial genomes and "missing" enzymes: redefining biochemical pathways. Arch Microbiol 172, 269-279.

Delorme, C., Godon, J.-J., Ehrlich, S. D. \& Renault, P. (1993). Gene inactivation in Lactococcus lactis: histidine biosynthesis. J Bacteriol 175, 4391-4399.

Edwards, C. J., Innes, D. J., Burns, D. M. \& Beacham, I. R. (1993). UDP-sugar hydrolase isozymes in Salmonella enterica and Escherichia coli: silent alleles of $u s h A$ in related strains of group I Salmonella isolates, and of $u s h B$ in wild-type and K12 strains of $E$. coli, indicate recent and early silencing events, respectively. FEMS Microbiol Lett 114, 293-298.

Feng, P. \& Lampel, K. A. (1994). Genetic analysis of uidA expression in enterohaemorrhagic Escherichia coli serotype O157: H7. Microbiology 140, 2101-2107.

Fraser, C. M., Norris, S. J., Weinstock, G. M. \& 22 other authors (1998). Complete genome sequence of Treponema pallidum, the syphilis spirochete. Science 281, 375-388.

Fraser, C. M., Eisen, J. A. \& Salzberg, S. L. (2000). Microbial genome sequencing. Nature 406, 799-803.

Garrett, A. R., Johnson, L. A. \& Beacham, I. R. (1989). Isolation, molecular characterization and expression of the $u s h B$ gene of Salmonella typhimurium which encodes a membrane-bound UDP-sugar hydrolase. Mol Microbiol 3, 177-186.

Glaser, L., Melo, A. \& Paul, R. (1967). Uridine diphosphate sugar hydrolase. Purification of enzyme and protein inhibitor. $\mathrm{J} \mathrm{Biol}$ Chem 242, 1944-1954.

Hall, B. G. (1981). Changes in the substrate specificities of an enzyme during directed evolution of new functions. Biochemistry 20, 4042-4049.
Hall, B. G. (1989). Selection, adaptation, and bacterial operons. Genome 31, 265-271.

Hall, B. G. \& Betts, P. W. (1987). Cryptic genes for cellobiose utilization in natural isolates of Escherichia coli. Genetics 115, 431-439.

Hall, B. G., Yokoyama, S. \& Calhoun, D. H. (1983). Role of cryptic genes in microbial evolution. Mol Biol Evol 1, 109-124.

Hartley, B. S. (1974). Enzyme families. In Evolution in the Microbial World, pp. 151-182. Edited by M. J. Carlile \& J. J. Skehel. Cambridge: Cambridge University Press.

Hartley, B. S. (1979). Evolution of enzyme structure. Proc R Soc Lond B Biol Sci 205, 443-452.

Hartley, B. S. (1980). Experimental enzyme evolution. In The Evolution of Protein Structure and Function, pp. 39-48. Edited by D. S. Sigman \& M. A. B. Brazier. New York: Academic Press.

Hartley, B. S (1984). Experimental evolution of ribitol dehydrogenase. In Microorganisms as Model Systems for Studying Evolution, pp. 23-54. Edited by R. P. Mortlock. New York: Plenum.

Henikoff, S. \& Henikoff, J. G. (1992). Amino acid substitution matrices from protein blocks. Proc Natl Acad Sci USA 89, 10915-10919.

Hill, C. W. \& Harnish, B. W. (1981). Inversions between ribosomal RNA genes of Escherichia coli. Proc Natl Acad Sci USA 78, 7069-7072.

Jones, R. A., Burns, D. M., Carruthers, D. J. \& Beacham, I. R. (1993). Membrane localisation of a UDP-sugar hydrolase, in Salmonella, is by an uncleaved N-terminal signal peptide. FEMS Microbiol Lett 114, 299-304.

Knöfel, T. \& Sträter, N. (1999). X-ray structure of the Escherichia coli periplasmic 5'-nucleotidase containing a dimetal catalytic site. Nature Struct Biol 6, 448-453.

Koch, A. L. (1972). Enzyme evolution. I. The importance of untranslatable intermediates. Genetics 72, 297-316.

Koonin, E. V. (1994). Conserved sequence pattern in a wide variety of phosphoesterases. Protein Sci 3, 356-358.

Kunkel, T. A. (1985). Rapid and efficient site-specific mutagenesis without phenotypic selection. Proc Natl Acad Sci USA 82, 488-492.

Labedan, B. \& Riley, M. (1995). Widespread protein sequence similarities: origins of Escherichia coli genes. J Bacteriol 177, 1585-1588.

Liyou, N., Hamilton, S., Elvin, C. \& Willadsen, P. (1999). Cloning and expression of ecto $5^{\prime}$-nucleotidase from the cattle tick Boophilus microplus. Insect Mol Biol 8, 257-266.

Mighell, A. J., Smith, N. R., Robinson, P. A. \& Markham, A. F. (2000). Vertebrate pseudogenes. FEBS Lett 468, 109-114.

Misumi, Y., Ogata, S., Ohkubo, K., Hirose, S. \& Ikehara, Y. (1990a). Primary structure of human placental 5'-nucleotidase and identification of the glycolipid anchor in the mature form. Eur J Biochem 191, 563-569.

Misumi, Y., Ogata, S., Hirose, S. \& Ikehara, Y. (1990b). Primary structure of rat liver $5^{\prime}$-nucleotidase deduced from the cDNA. Presence of the $\mathrm{COOH}$-terminal hydrophobic domain for possible post-translational modification by glycophospholipid. J Biol Chem 265, 2178-2183.

Nakamaye, K. L. \& Eckstein, F. (1986). Inhibition of restriction endonuclease Ncil cleavage by phosphorothioate groups and its application to oligonucleotide-directed mutagenesis. Nucleic Acids Res 14, 9679-9698.

Neu, H. C. (1968). The $5^{\prime}$-nucleotidases and cyclic phospho- 
diesterases (3'-nucleotidases) of the Enterobacteriaceae. J Bacteriol 95, 1732-1737.

Pollack, J. D. (1997). Mycoplasma genes: a case for reflective annotation. Trends Microbiol 5, 413-418.

Reeves, M. W., Evins, G. M., Heiba, A. A., Plikaytis, B. D. \& Farmer, J. J. (1989). Clonal nature of Salmonella typhi and its genetic relatedness to other salmonellae as shown by multilocus enzyme electrophoresis, and proposal of Salmonella bongori comb. nov. J Clin Microbiol 27, 313-320.

Resta, R., Hooker, S. W., Hansen, K. R., Laurent, A. B., Park, J. L., Blackburn, M. R., Knudsen, T. B. \& Thompson, L. F. (1993). Murine ecto-5'-nucleotidase (CD73): cDNA cloning and tissue distribution. Gene 133, 171-177.

Rigby, P. W. J., Burleigh, B. D. \& Hartley, B. S. (1974). Gene duplication in experimental enzyme evolution. Nature 251, 200-204.

Riley, M. \& Labedan, B. (1997). Protein evolution viewed through Escherichia coli protein sequences: introducing the notion of a structural segment of homology, the module. J Mol Biol 268, 857-868.

Riley, M. \& Sanderson, K. E. (1990). Comparative genetics of Escherichia coli and Salmonella typhimurium. In The Bacterial Chromosome, pp. 85-95. Edited by K. Drlica \& M. Riley. Washington, DC: American Society for Microbiology.

Rine, J. (1999). On the mechanism of silencing in Escherichia coli. Proc Natl Acad Sci US A 96, 8309-8311.

Steed, P. M. \& Wanner, B. L. (1993). Use of the rep technique for allele replacement to construct mutants with deletions of the
pstSCAB-phoU operon: evidence of a new role for the PhoU protein in the phosphate regulon. J Bacteriol 175, 6797-6809.

Suzuki, K., Furukawa, Y., Tamura, H., Ejiri, N., Suematsu, H., Taguchi, R., Nakamura, S., Suzuki, Y. \& Ikezawa, H. (1993). Purification and cDNA cloning of bovine liver $5^{\prime}$-nucleotidase, a GPI-anchored protein, and its expression in COS cells. J Biochem $113,607-613$.

Tamao, Y., Noguchi, K., Sakai-Tomita, Y., Hama, H., Shimamoto, T., Kanazawa, H., Tsuda, M. \& Tsuchiya, T. (1991). Sequence analysis of nutA gene encoding membrane-bound $\mathrm{Cl}^{-}$-dependent 5 '-nucleotidase of Vibrio parahaemolyticus. J Biochem 109, 24-29.

Taylor, J. W., Ott, J. \& Eckstein, F. (1985). The rapid generation of oligonucleotide-directed mutations at high frequency using phosphorothioate-modified DNA. Nucleic Acids Res 13, 8765-8785.

Thompson, J. D., Higgins, D. G. \& Gibson, T. J. (1994). CLUSTAL W : improving the sensitivity of progressive multiple sequence alignment through sequence weighting, position-specific gap penalties and weight matrix choice. Nucleic Acids Res 22, 4673-4680.

Volknandt, W., Vogel, M., Pevsner, J., Misumi, Y., Ikehara, Y. \& Zimmermann, H. (1991). 5'-nucleotidase from the electric ray electric lobe. Primary structure and relation to mammalian and procaryotic enzymes. Eur J Biochem 202, 855-861.

Zimmermann, H. (1992). 5'-Nucleotidase: molecular structure and functional aspects. Biochem J 285, 345-365.

Received 29 December 2000; revised 6 March 2001; accepted 22 March 2001. 